Article

\title{
Evolutionary Machine Learning for Optimal Polar-Space Fuzzy Control of Cyber-Physical Mecanum Vehicles
}

\author{
Hsu-Chih Huang * and Jing-Jun Xu \\ Department of Electrical Engineering, National Ilan University, Yilan 260007, Taiwan; r0841013@ms.niu.edu.tw \\ * Correspondence: hchuang@niu.edu.tw
}

Received: 13 October 2020; Accepted: 16 November 2020; Published: 18 November 2020

check for updates

\begin{abstract}
This paper contributes to the development of evolutionary machine learning (EML) for optimal polar-space fuzzy control of cyber-physical Mecanum vehicles using the flower pollination algorithm (FPA). The metaheuristic FPA is utilized to design optimal fuzzy systems, called FPA-fuzzy. In this hybrid computation, both the fuzzy structure and the number of IF-THEN rules are optimized through the FPA evolutionary process. This approach overcomes the drawback of the structure tuning problem in conventional fuzzy systems. After deriving the polar-space kinematics model of Mecanum vehicles, an optimal EML FPA-fuzzy online control scheme is synthesized, and the global stability is proven via Lyapunov theory. An embedded cyber-physical robotic system is then constructed using the typical 5C strategy. The proposed FPA-fuzzy computation collaborates with the advanced sensors and actuators of Mecanum vehicles to design a cyber-physical robotic system. Compared with conventional Cartesian-space control methods, the proposed EML FPA-fuzzy has the advantages of metaheuristics, fuzzy online control, and cyber-physical system design in polar coordinates. Finally, the mechatronic design and experimental setup of a Mecanum vehicle cyber-physical system is constructed. Through experimental results and comparative works, the effectiveness and merit of the proposed methods are validated. The proposed EML FPA-fuzzy control approach has theoretical and practice significance in terms of its real-time capability, online parameter tuning, convergent behavior, and hybrid artificial intelligence.
\end{abstract}

Keywords: evolutionary; machine learning; cyber-physical system; fuzzy control; Mecanum vehicles; flower pollination algorithm

\section{Introduction}

Evolutionary machine learning (EML) has been regarded the most promising and challenging approach to the next stage of artificial intelligence (AI) [1-5]. Recently, this fast-growing field has been extensively applied in many fields with great success, including computer vision, combinatorial optimization, medical imaging, bioinformatics, and text analytics [4,5]. This evolutionary computation is a sub-field of AI that comprises a family of nature-inspired algorithms. The population-based algorithms maintain a population of candidate solutions and evolve towards the global optimum. Having natural evolution and biological genetics, EML has been widely employed to solve optimization problems in high-dimensional search spaces systematically and efficiently. This approach offers a number of exclusive advantages, including robustness, reliable performance, flexibility, and global search capability. Consequently, there have been many EML studies related to engineering optimization using evolutionary algorithms, such as the genetic algorithm (GA), artificial bee colony algorithm $(\mathrm{ABC})$, bat algorithm (BA), and particle swarm optimization (PSO) [1-6]. 
In recent years, many EMLs have been presented to address real-world optimization problems [7-9]. Among them, the flower pollination algorithm (FPA), developed by Yang in 2012, is a swarm intelligence technology that mimics the pollination behavior of flowering plants [10]. In nature, there are two main types of pollination for flowers: biotic pollination (cross-pollination) and abiotic (self-pollination). About $90 \%$ of flowering plants depend on biotic pollination, in which the pollen is transferred by pollinators such as insects and animals. Abiotic pollination does not does not require any pollinators; the wind behaves as the pollen spreader between adjacent flowers. This form of EML has attracted considerable attention in industry and academic optimization fields due to its impressive characteristics [11]. Some researchers have proved that this metaheuristic is superior to traditional evolutionary algorithms in terms of convergence velocity, optimization precision, and local optimum avoidance. Therefore, this new biological EML algorithm has shown robust performance when applied to solving various real-world optimization problems [10,11].

Fuzzy control is a pragmatic engineering approach for a variety of challenging control applications [12-14]. It provides a formal methodology for representing, manipulating, and implementing a human's heuristic knowledge to control a system. A typical fuzzy system is composed of four main parts: fuzzification, a knowledge base (IF-THEN rules), a fuzzy inference engine, and defuzzification [15]. In fuzzy control systems, the fuzzifier maps a real crisp input to a fuzzy function, and the "IF-THEN" rules comprise the knowledge base of the application domain $[15,16]$. The inference engine provides the decision-making logic of the controller, and the defuzzification process converts fuzzy control values into crisp quantities. This study presents an optimal polar-space EML fuzzy control method for cyber-physical Mecanum vehicles using FPA computation.

Structure optimization is a major challenge when applying fuzzy-based configurations efficiently $[17,18]$. The rule base and membership functions influence the performance of fuzzy control systems. To address this problem, there have been studies on training fuzzy architectures and techniques to improve accuracy and performance in recent years [19-21]. However, these techniques are computationally expensive and may become trapped in local optima [22]. In this paper, an embedded FPA-optimized fuzzy control scheme is presented to avoid these disadvantages. To the authors' best understanding, there has been no previous attempt to develop embedded FPA-fuzzy control methods for autonomous mobile vehicles.

Most evolutionary learning control systems are realized by means of a modern central processor unit (CPU) that takes huge computation and memory resources [23]. Nowadays, embedded metaheuristics is an emerging technology for deploying EML by considering power management and high-performance inferences for real-time applications [24,25]. Thanks to the development of power-efficient embedded computing platforms, this approach has become a cost-effective alternative for designing advanced EML fuzzy cyber-physical systems by including sensors and actuators. The proposed FPA-fuzzy EML is realized in a field-programmable gate array (FPGA) embedded processor incorporated with custom logic components to construct inexpensive optimal cyber-physical systems for mobile vehicles using the typical 5C architecture [24-26].

Embedded systems design with FPGAs is increasingly becoming a dominant methodology in modern electronic design [26,27]. An FPGA contains a matrix of reconfigurable gate array logic circuitry that can be programmed and configured in the field. Having the advantages of flexibility and reconfigurability, this approach has been widely used in designing sophisticated cyber-physical embedded systems, ranging from intelligent power systems to robot control, manufacturing systems, and security systems. These embedded systems integrate computational and physical processes by including connected sensors and actuators to accomplish closed-loop feedback control in one FPGA chip [28]. The FPGA-based EML optimal fuzzy control of cyber-physical systems for Mecanum vehicles deserves further investigation.

A Mecanum vehicle is a type of mobile robot equipped with four Mecanum omnidirectional wheels. The passive rollers are mounted around the circumference of the wheel at 45 degrees to the wheel plane $[29,30]$. In comparison with the traditional differential-drive and omnidirectional mobile 
robots, these Mecanum vehicles have been shown more to be effective in achieving omnidirectional capability [30]. This kind of omnidirectional mobile vehicle is particularly useful in designing automated guided vehicles, intelligent wheelchairs, mobile manipulators, etc. The mathematical modeling and controller design of Mecanum vehicles have been investigated by several researchers [29-31]. Generally speaking, the vehicle model has been derived, and the redundant control problem has been addressed as well [29-31]. However, all the studies of Mecanum vehicles have employed Cartesian space to cope with the modeling and controller design problems.

In comparison to the Cartesian-space approach, polar-space control and modeling for mobile robots has been proven more efficient in tracking some special trajectories, such as the limacon of Pascal, rose curves, and cardioid curves [32-34]. Polar-space controller design and model analysis of the classical nonholonomic vehicles was addressed in reference [32]. Some researchers investigated the tracking controller design of traditional omnidirectional mobile vehicles in polar coordinates [33,34]. These works clearly indicate the effectiveness and merits of the polar-space control and modeling of mobile robotics. However, EML for optimal polar-space fuzzy control of cyber-physical Mecanum vehicles using FPA remains open.

The rest of this paper is organized as follows. Section 2 presents the mathematical kinematics modeling and tracking control of Mecanum vehicles in polar coordinates. Both the kinematic control problem and stability analysis are investigated. Section 3 elaborates the FPA-fuzzy hybrid computation and its application to optimal online control of Mecanum vehicles. Based on the typical 5C architecture, an embedded cyber-physical robotic system is constructed by including robotic sensors and actuators. The experimental results, comparative works, and discussion are provided in Section 4 to demonstrate the effectiveness and merit of the proposed methodologies. Section 5 concludes this paper.

\section{Polar-Space Modeling and Tracking Control of Mecanum Mobile Robots}

Figure 1 presents the geometrical structure of the four-wheeled Mecanum mobile robots. Due to the four driving degrees of freedom (DOFs), the kinematics model is categorized as a redundancy problem. This section aims at deriving the polar-space kinematics model of Mecanum vehicles, thus resolving the polar redundancy problem. Both the forward and inverse kinematics are investigated in polar coordinates. With the polar-space kinematics model of Mecanum vehicles, a tracking control scheme is synthesized, and the stability is proven via Lyapunov stability theory.

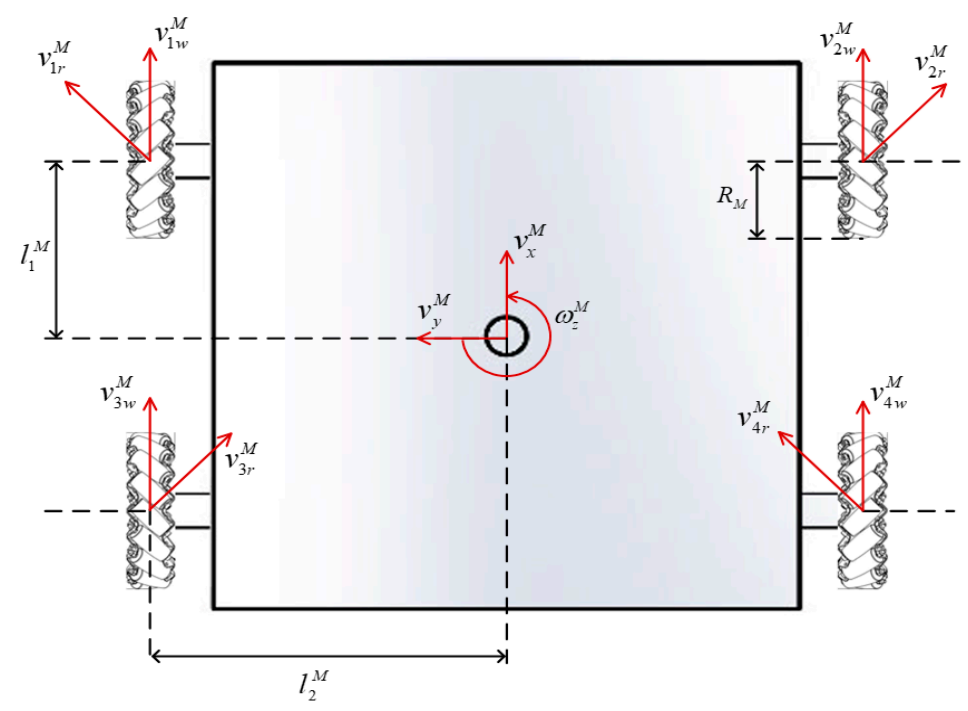

Figure 1. Geometrical structure of the Mecanum vehicles. 


\subsection{Polar-Space Kinematics Analysis}

In mobile robotics, kinematics analysis can be classified into Cartesian coordinates and polar coordinates. The kinematics equations are very important in designing motion controllers. Cartesian-space kinematics analysis considering the four-wheeled Mecanum vehicle's geometrical configuration is summarized by

$$
V_{W}^{M}=\left[\begin{array}{ccc}
1 & -1 & -\left(l_{1}^{M}+l_{2}^{M}\right) \\
1 & 1 & \left(l_{1}^{M}+l_{2}^{M}\right) \\
1 & 1 & -\left(l_{1}^{M}+l_{2}^{M}\right) \\
1 & -1 & \left(l_{1}^{M}+l_{2}^{M}\right)
\end{array}\right] V_{C}^{M}=T_{C}^{M} V_{C}^{M}
$$

where $V_{W}^{M}=\left[\begin{array}{llll}v_{1 w}^{M} & v_{2 w}^{M} & v_{3 w}^{M} & v_{4 w}^{M}\end{array}\right]^{T}$ is the velocity vector of the four Mecanum omnidirectional wheels with passive rollers. $V_{C}^{M}=\left[\begin{array}{lll}v_{x}^{M} & v_{y}^{M} & \omega_{z}^{M}\end{array}\right]^{T}$ represents the vehicle's velocity vector in Cartesian coordinates. Based on the inverse kinematic equation in Equation (1), the forward kinematic equation can be derived using a pseudo inverse matrix approach, expressed by

$$
\left[\begin{array}{c}
v_{x}^{M} \\
v_{y}^{M} \\
\omega_{z}^{M}
\end{array}\right]=\frac{1}{4}\left[\begin{array}{cccc}
1 & 1 & 1 & 1 \\
-1 & 1 & 1 & -1 \\
\frac{-1}{\left(l_{1}^{M}+l_{2}^{M}\right)} & \frac{1}{\left(l_{1}^{M}+l_{2}^{M}\right)} & \frac{-1}{\left(l_{1}^{M}+l_{2}^{M}\right)} & \frac{1}{\left(l_{1}^{M}+l_{2}^{M}\right)}
\end{array}\right]\left[\begin{array}{c}
R_{M} \dot{\theta}_{1} \\
R_{M} \dot{\theta}_{2} \\
R_{M} \dot{\theta}_{3} \\
R_{M} \dot{\theta}_{4}
\end{array}\right]
$$

where $v_{i w}^{M}=R_{M} \dot{\theta}_{i}, i=1,2,3,4$, and $R_{M}$ is the Mecanum wheels' radius. $l_{1}^{M}$ and $l_{2}^{M}$ are the width and length of the vehicle shown in Figure 1. Both the Cartesian-space forward and inverse kinematic equations of Mecanum vehicles in a moving frame are presented. Considering the Mecanum vehicle's position and orientation $\left[\begin{array}{lll}x_{M} & y_{M} & \theta_{M}\end{array}\right]^{T}$ in the world frame, the Cartesian-space forward kinematics equation is formulated as follows:

$$
\begin{aligned}
& {\left[\begin{array}{c}
\dot{x}_{M} \\
\dot{y}_{M} \\
\dot{\theta}_{M}
\end{array}\right]=\frac{1}{4}\left[\begin{array}{cccc}
\left(\cos \theta_{M}+\sin \theta_{M}\right) & \left(\cos \theta_{M}-\sin \theta_{M}\right) & \left(\cos \theta_{M}-\sin \theta_{M}\right) & \left(\cos \theta_{M}+\sin \theta_{M}\right) \\
-\left(\cos \theta_{M}-\sin \theta_{M}\right) & \left(\cos \theta_{M}+\sin \theta_{M}\right) & \left(\cos \theta_{M}+\sin \theta_{M}\right) & -\left(\cos \theta_{M}-\sin \theta_{M}\right) \\
\frac{-1}{\left(1_{1}^{M}+l_{2}^{M}\right)} & \frac{1}{\left(l_{1}^{M}+l_{2}^{M}\right)} & \frac{-1}{\left(l_{1}^{M}+l_{2}^{M}\right)} & \frac{1}{\left(l_{1}^{M}+l_{2}^{M}\right)}
\end{array}\right]\left[\begin{array}{c}
v_{1 w}^{M} \\
v_{2 w}^{M} \\
v_{3 w}^{M} \\
v_{4 w}^{M}
\end{array}\right]} \\
& =\frac{1}{4}\left[\begin{array}{cccc}
\sqrt{2} \sin \vartheta_{M} & \sqrt{2} \cos \vartheta_{M} & \sqrt{2} \cos \vartheta_{M} & \sqrt{2} \sin \vartheta_{M} \\
-\sqrt{2} \cos \vartheta_{M} & \sqrt{2} \sin \vartheta_{M} & \sqrt{2} \sin \vartheta_{M} & -\sqrt{2} \cos \vartheta_{M} \\
\frac{-1}{\left(l_{1}^{M}+l_{2}^{M}\right)} & \frac{1}{\left(l_{1}^{M}+l_{2}^{M}\right)} & \frac{-1}{\left(l_{1}^{M}+l_{2}^{M}\right)} & \frac{1}{\left(l_{1}^{M}+l_{2}^{M}\right)}
\end{array}\right]\left[\begin{array}{c}
v_{1 w}^{M} \\
v_{2 w w}^{M} \\
v_{3 w}^{M} \\
v_{4 w}^{M}
\end{array}\right]=P_{M}^{+}\left(\theta_{M}\right)\left[\begin{array}{c}
v_{1 w}^{M} \\
v_{2 w}^{M} \\
v_{3 w}^{M} \\
v_{4 w}^{M}
\end{array}\right]
\end{aligned}
$$

where $\vartheta_{M}=\theta_{M}+\frac{\pi}{4}$. Combining Equations (1)-(3), the following summarizes the inverse kinematics equation of the four-wheeled Mecanum platform in Cartesian space:

$$
\left[\begin{array}{c}
v_{1 w}^{M} \\
v_{2 w}^{M} \\
v_{3 w}^{M} \\
v_{4 w}^{M}
\end{array}\right]=\left[\begin{array}{ccc}
\sqrt{2} \sin \left(\vartheta_{M}\right) & -\sqrt{2} \cos \left(\vartheta_{M}\right) & -\left(l_{1}^{M}+l_{2}^{M}\right) \\
\sqrt{2} \cos \left(\vartheta_{M}\right) & \sqrt{2} \sin \left(\vartheta_{M}\right) & \left(l_{1}^{M}+l_{2}^{M}\right) \\
\sqrt{2} \cos \left(\vartheta_{M}\right) & \sqrt{2} \sin \left(\vartheta_{M}\right) & -\left(l_{1}^{M}+l_{2}^{M}\right) \\
\sqrt{2} \sin \left(\vartheta_{M}\right) & -\sqrt{2} \cos \left(\vartheta_{M}\right) & \left(l_{1}^{M}+l_{2}^{M}\right)
\end{array}\right]\left[\begin{array}{c}
\dot{x}_{M} \\
\dot{y}_{M} \\
\dot{\theta}_{M}
\end{array}\right]=P_{M}(\theta)\left[\begin{array}{c}
\dot{x}_{M} \\
\dot{y}_{M} \\
\dot{\theta}_{M}
\end{array}\right]
$$

where $P_{M}^{+}(\theta)$ is the left pseudo inverse matrix of $P_{M}\left(\theta_{M}\right)$.

In order to derive the polar-space model of Mecanum vehicles, one applies the following relationship:

$$
r_{M}(t)=\sqrt{x_{M}^{2}(t)+y_{M}^{2}(t)}, x_{M}(t)=r_{M}(t) \cos \varphi_{M}(t), y_{M}(t)=r_{M}(t) \sin \varphi_{M}(t)
$$

and their time derivatives 


$$
\begin{aligned}
& \dot{r}_{M}(t)=\frac{x_{M}(t) \dot{x}_{M}(t)+y_{M}(t) \dot{y}_{M}(t)}{\sqrt{x_{M}^{2}(t)+y_{M}^{2}(t)}}=\frac{x_{M}(t) \dot{x}_{M}(t)+y_{M}(t) \dot{y}_{M}(t)}{r_{M}(t)} \\
& \dot{x}_{M}(t)=\dot{r}_{M}(t) \cos \varphi_{M}(t)-r_{M}(t) \dot{\varphi}_{M}(t) \sin \varphi_{M}(t) \\
& \dot{y}_{M}(t)=\dot{r}_{M}(t) \sin \varphi_{M}(t)+r_{M}(t) \dot{\varphi}_{M}(t) \cos \varphi_{M}(t)
\end{aligned}
$$

where $r_{M}$ denotes the polar radius and $\varphi_{M}$ is the polar angle. Combining Equations (5) and (6), one obtains

$$
\begin{aligned}
& \dot{r}_{M}(t)=\frac{x_{M}(t) \dot{x}_{M}(t)+y_{M}(t) \dot{y}_{M}(t)}{r_{M}^{(t)}}=\frac{\dot{x}_{M}(t) r_{M}(t) \cos \varphi_{M}(t)+\dot{y}_{M}(t) r_{M}(t) \sin \varphi_{M}(t)}{r_{M}(t)} \\
& =\dot{x}_{M}(t) \cos \varphi_{M}(t)+\dot{y}_{M}(t) \sin \varphi_{M}(t) \\
& =\left[\begin{array}{llll}
\frac{1}{2 \sqrt{2}} \sin \left(\vartheta_{M}(t)-\varphi_{M}(t)\right) & \frac{1}{2 \sqrt{2}} \cos \left(\vartheta_{M}(t)-\varphi_{M}(t)\right) & \frac{1}{2 \sqrt{2}} \cos \left(\vartheta_{M}(t)-\varphi_{M}(t)\right) & \frac{1}{2 \sqrt{2}} \sin \left(\vartheta_{M}(t)-\varphi_{M}(t)\right)
\end{array}\right]\left[\begin{array}{c}
R_{M} \omega_{1}(t) \\
R_{M} \omega_{2}(t) \\
R_{M} \omega_{3}(t) \\
R_{M} \omega_{4}(t)
\end{array}\right] .
\end{aligned}
$$

Moreover, taking the following equality into Equation (6), one obtains

$$
\begin{aligned}
& \dot{y}_{M}(t) \cos \varphi_{M}(t)-\dot{x}_{M}(t) \sin \varphi_{M}(t)=r_{M}(t)\left(\sin ^{2} \varphi_{M}(t)+\cos ^{2} \varphi_{M}(t)\right) \dot{\varphi}_{M}(t)=r_{M}(t) \dot{\varphi}_{M}(t) \\
& \dot{\varphi}_{M}(t)=\frac{1}{r_{M}(t)}\left(\dot{y}_{M}(t) \cos \varphi_{M}(t)-\dot{x}_{M}(t) \sin \varphi_{M}(t)\right)
\end{aligned}
$$



From Equation (4),

$$
\dot{\theta}_{M}(t)=\left[\begin{array}{llll}
\frac{-1}{4\left(l_{1}^{M}+l_{2}^{M}\right)} & \frac{1}{4\left(l_{1}^{M}+l_{2}^{M}\right)} & \frac{-1}{4\left(l_{1}^{M}+l_{2}^{M}\right)} & \frac{1}{4\left(l_{1}^{M}+l_{2}^{M}\right)}
\end{array}\right]\left[\begin{array}{l}
R_{M} \omega_{1}(t) \\
R_{M} \omega_{2}(t) \\
R_{M} \omega_{3}(t) \\
R_{M} \omega_{4}(t)
\end{array}\right]
$$

Combining Equations (7)-(9), the polar-space kinematics model of the redundant Mecanum vehicles is described as follows:

$$
\left[\begin{array}{c}
\dot{r}_{M}(t) \\
\dot{\varphi}_{M}(t) \\
\dot{\theta}_{M}(t)
\end{array}\right]=T_{M}^{\#}\left(r_{M}(t), \vartheta_{M}(t)-\varphi_{M}(t)\right)\left[\begin{array}{c}
R_{M} \omega_{1}(t) \\
R_{M} \omega_{2}(t) \\
R_{M} \omega_{3}(t) \\
R_{M} \omega_{4}(t)
\end{array}\right]
$$

where

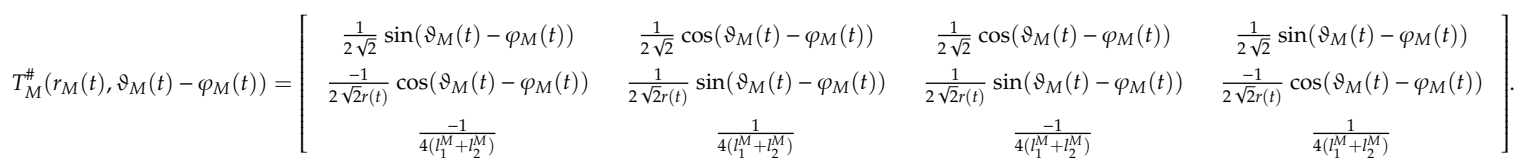

The pseudo inverse matrix of $T_{M}^{\#}\left(r_{M}(t), \vartheta_{M}(t)-\varphi_{M}(t)\right)$ is given by

$$
T_{M}\left(r_{M}(t), \vartheta_{M}(t)-\varphi_{M}(t)\right)=\left[\begin{array}{ccc}
\sqrt{2} \sin \left(\vartheta_{M}(t)-\varphi_{M}(t)\right) & -\sqrt{2} r_{M}(t) \cos \left(\vartheta_{M}(t)-\varphi_{M}(t)\right) & -\left(l_{1}^{M}+l_{2}^{M}\right) \\
\sqrt{2} \cos \left(\vartheta_{M}(t)-\varphi_{M}(t)\right) & \sqrt{2} r_{M}(t) \sin \left(\vartheta_{M}(t)-\varphi_{M}(t)\right) & \left(l_{1}^{M}+l_{2}^{M}\right) \\
\sqrt{2} \cos \left(\vartheta_{M}(t)-\varphi_{M}(t)\right) & \sqrt{2} r_{M}(t) \sin \left(\vartheta_{M}(t)-\varphi_{M}(t)\right) & -\left(l_{1}^{M}+l_{2}^{M}\right) \\
\sqrt{2} \sin \left(\vartheta_{M}(t)-\varphi_{M}(t)\right) & -\sqrt{2} r_{M}(t) \cos \left(\vartheta_{M}(t)-\varphi_{M}(t)\right) & \left(l_{1}^{M}+l_{2}^{M}\right)
\end{array}\right]
$$




\subsection{Polar-Space Tracking Control}

Tracking control is regarded as a challenging problem in mobile robotics research. It is concerned with controller design to drive wheeled robots to track a time-varying geometric trajectory. This subsection is devoted to developing a polar-space tracking control law to achieve this trajectory tracking task. Having the kinematics model in polar coordinates, the next goal is to synthesize a polar-space motion control scheme to accomplish trajectory tracking. More precisely, the controller automatically generates the velocity command vector $\left[\begin{array}{cccc}v_{1 w}^{M} & v_{2 w}^{M} & v_{3 w}^{M} & v_{4 w}^{M}\end{array}\right]^{T}$ to steer the Mecanum redundant vehicle from any initial position and orientation $\left[\begin{array}{ccc}r_{0}^{M} & \varphi_{0}^{M} & \theta_{0}^{M}\end{array}\right]^{T}$ to follow any smooth and differentiable polar-space trajectory $\left[\begin{array}{llll}r_{d}^{M}(t) & \varphi_{d}^{M}(t) & \theta_{d}^{M}(t)\end{array}\right]^{T}$. The current pose that includes position and orientation at time $t$ is represented by $\left[\begin{array}{llll}r_{M}(t) & \varphi_{M}(t) & \theta_{M}(t)\end{array}\right]^{T}$, and the tracking error in polar coordinates is then described by

$$
\left[\begin{array}{c}
r_{e}^{M}(t) \\
\varphi_{e}^{M}(t) \\
\theta_{e}^{M}(t)
\end{array}\right]=\left[\begin{array}{c}
r_{M}(t) \\
\varphi_{M}(t) \\
\theta_{M}(t)
\end{array}\right]-\left[\begin{array}{c}
r_{d}^{M}(t) \\
\varphi_{d}^{M}(t) \\
\theta_{d}^{M}(t)
\end{array}\right]
$$

Differentiating Equation (12), the polar-space closed-loop error system is formulated by

$$
\left[\begin{array}{c}
\dot{r}_{e}^{M}(t) \\
\dot{\varphi}_{e}^{M}(t) \\
\dot{\theta}_{e}^{M}(t)
\end{array}\right]=T_{M}^{\#}\left(r_{M}(t), \varphi_{M}(t)-\theta_{M}(t)\right)\left[\begin{array}{c}
R_{M} \omega_{1}(t) \\
R_{M} \omega_{2}(t) \\
R_{M} \omega_{3}(t) \\
R_{M} \omega_{4}(t)
\end{array}\right]-\left[\begin{array}{c}
\dot{r}_{d}^{M}(t) \\
\dot{\varphi}_{d}^{M}(t) \\
\dot{\theta}_{d}^{M}(t)
\end{array}\right]
$$

Considering the closed-loop Equation (13) that includes the kinematics model in polar coordinates, the next goal is to synthesize a control law to determine $\left[\begin{array}{cccc}\omega_{1}(t) & \omega_{2}(t) & \omega_{3}(t) & \omega_{4}(t)\end{array}\right]^{T}$, thereby making the Mecanum robotic system asymptotically stable. In doing so, the following polar-space control law is proposed to accomplish the trajectory tracking task. Notice that the proportional-integral-differential (PID) control strategy is included in this subsection because it is the most widely applied feedback control algorithm.

$$
\left[\begin{array}{c}
\omega_{1}(t) \\
\omega_{2}(t) \\
\omega_{3}(t) \\
\omega_{4}(t)
\end{array}\right]=\frac{1}{R_{M}} T_{M}\left(r_{M}(t), \theta_{M}(t)-\varphi_{M}(t)\right)\left(-K_{p}\left[\begin{array}{c}
r_{e}^{M}(t) \\
\varphi_{e}^{M}(t) \\
\theta_{e}^{M}(t)
\end{array}\right]-K_{I}\left[\begin{array}{c}
\int_{0}^{t} r_{e}^{M}(\tau) d \tau \\
\int_{0}^{t} \varphi_{e}^{M}(\tau) d \tau \\
\int_{0}^{t} \theta_{e}^{M}(\tau) d \tau
\end{array}\right]+\left[\begin{array}{c}
\dot{r}_{d}^{M}(t) \\
\dot{\varphi}_{d}^{M}(t) \\
\dot{\theta}_{e}^{M}(t)
\end{array}\right]\right)
$$

Here, $K_{P}$ and $K_{I}$ are gain matrices in the proposed control law. These control gain matrices are symmetric and positive definite, that is, $K_{P}=\operatorname{diag}\left\{K_{p 1}, K_{p 2}, K_{p 3}\right\}=K_{P}{ }^{T}>0$ and $K_{I}=\operatorname{diag}\left\{K_{i 1}, K_{i 2}, K_{i 3}\right\}=K_{I}^{T}>0$. Substituting Equation (14) into Equation (13), the closed-loop error of the Mecanum vehicle system is described as follows.

$$
\left[\begin{array}{c}
\dot{r}_{e}^{M}(t) \\
\dot{\varphi}_{e}^{M}(t) \\
\dot{\theta}_{e}^{M}(t)
\end{array}\right]=-K_{p}\left[\begin{array}{c}
r_{e}^{M}(t) \\
\varphi_{e}^{M}(t) \\
\theta_{e}^{M}(t)
\end{array}\right]-K_{I}\left[\begin{array}{c}
\int_{0}^{t} r_{e}^{M}(\tau) d \tau \\
\int_{0}^{t} \varphi_{e}^{M}(\tau) d \tau \\
\int_{0}^{t} \theta_{e}^{M}(\tau) d \tau
\end{array}\right]
$$

The globally asymptotical stability of the closed-loop system in Equation (15) is proved herein using the well-known Lyapunov stability theory. Based on this theory, the following Lyapunov function was selected to prove the system stability. 


$$
V(t)=\frac{1}{2}\left[\begin{array}{lll}
r_{e}^{M}(t) & \varphi_{e}^{M}(t) & \theta_{e}^{M}(t)
\end{array}\right]\left[\begin{array}{c}
r_{e}^{M}(t) \\
\varphi_{e}^{M}(t) \\
\theta_{e}^{M}(t)
\end{array}\right]+\frac{1}{2}\left[\begin{array}{lll}
\int_{0}^{t} r_{e}^{M}(\tau) d \tau & \int_{0}^{t} \varphi_{e}^{M}(\tau) d \tau & \int_{0}^{t} \theta_{e}^{M}(\tau) d \tau
\end{array}\right] K_{I}\left[\begin{array}{c}
\int_{0}^{t} r_{e}^{M}(\tau) d \tau \\
\int_{0}^{t} \varphi_{e}^{M}(\tau) d \tau \\
\int_{0}^{t} \theta_{e}^{M}(\tau) d \tau
\end{array}\right]
$$

The time derivative of $V(t)$ is described by

$$
\begin{aligned}
& \dot{V}(t)=\left[\begin{array}{lll}
r_{e}^{M}(t) & \varphi_{e}^{M}(t) & \theta_{e}^{M}(t)
\end{array}\right]\left[\begin{array}{c}
\dot{r}_{e}^{M}(t) \\
\dot{\varphi}_{e}^{M}(t) \\
\dot{\theta}_{e}^{M}(t)
\end{array}\right]+\left[\begin{array}{lll}
\int_{0}^{t} r_{e}^{M}(\tau) d \tau & \int_{0}^{t} \varphi_{e}^{M}(\tau) d \tau & \int_{0}^{t} \theta_{e}^{M}(\tau) d \tau
\end{array}\right] K_{I}\left[\begin{array}{c}
r_{e}^{M}(t) \\
\varphi_{e}^{M}(t) \\
\theta_{e}^{M}(t)
\end{array}\right] \\
& =\left[\begin{array}{lll}
r_{e}^{M}(t) & \varphi_{e}^{M}(t) & \theta_{e}^{M}(t)
\end{array}\right]-\left(K_{p}\left[\begin{array}{c}
r_{e}^{M}(t) \\
\varphi_{e}^{M}(t) \\
\theta_{e}^{M}(t)
\end{array}\right]-K_{I}\left[\begin{array}{c}
\int_{0}^{t} r_{e}^{M}(\tau) d \tau \\
\int_{0}^{t} \varphi_{e}^{M}(\tau) d \tau \\
\int_{0}^{t} \theta_{e}^{M}(\tau) d \tau
\end{array}\right]\right)+\left[\begin{array}{lll}
\int_{0}^{t} r_{e}^{M}(\tau) d \tau & \int_{0}^{t} \varphi_{e}^{M}(\tau) d \tau & \int_{0}^{t} \theta_{e}^{M}(\tau) d \tau
\end{array}\right] K_{I}\left[\begin{array}{c}
r_{e}^{M}(t) \\
\varphi_{e}^{M}(t) \\
\theta_{e}^{M}(t)
\end{array}\right] \\
& =-\left[\begin{array}{lll}
r_{e}^{M}(t) & \varphi_{e}^{M}(t) & \theta_{e}^{M}(t)
\end{array}\right] K_{p}\left[\begin{array}{c}
r_{e}^{M}(t) \\
\varphi_{e}^{M}(t) \\
\theta_{e}^{M}(t)
\end{array}\right] \leq 0
\end{aligned}
$$

This result implies that the position error and orientation error of the Mecanum vehicles converge to zero as time tends to infinity, that is, $\left[\begin{array}{lll}r_{e}^{M}(t) & \varphi_{e}^{M}(t) & \theta_{e}^{M}(t)\end{array}\right]^{T} \rightarrow\left[\begin{array}{lll}0 & 0 & 0\end{array}\right]^{T}$ as $t \rightarrow \infty$. The global stability of the proposed control law in Equation (14) for redundant four-wheeled Mecanum mobile vehicles is therefore proved.

\section{Polar-Space FPA-Fuzzy EML Control of Mecanum Vehicles}

\subsection{Flower Pollination Algorithm}

The pollination process is achieved through cross-pollination and self-pollination. Some birds behave as global pollinators to transfer pollen in cross-pollination. On the other hand, self-pollination is a process where pollens from the same flower are responsible for the fertilization process [10]. Biotic and cross-pollination are considered as global pollination processes performing Lévy flight. FPA abiotic and self-pollination behave as local pollination. These processes accomplish FPA exploration and exploitation in the multi-dimensional search space. In FPA, Lévy flight is a random walk operator based on the Lévy distribution, formulated by

$$
\operatorname{Levy}(\beta)=\left|\frac{\Gamma(\beta+1) \sin \left(\frac{\pi \beta}{2}\right)}{\Gamma\left(\frac{1+\beta}{2}\right) \beta(2)^{\left(\frac{\beta-1}{2}\right)}}\right|^{\frac{1}{\beta}}
$$

where $\Gamma$ is the gamma function and $\beta$ is a constant.

In the global pollination phase, flower pollens are carried by pollinators (insects) to travel over a long distance. This step ensures FPA pollination and reproduction of the fittest, $g_{\text {best }}$. The FPA flower constancy is expressed as follows:

$$
x_{i}^{t+1}=x_{i}^{t}+\gamma \operatorname{Levy}(\beta)\left(g_{\text {best }}-x_{i}^{t}\right)
$$

where $x_{i}^{t}$ is the $i$ th solution at the $t$ th iteration, and $g_{b e s t}$ is the best found solution. Step sizes are drawn randomly from the Lévy distribution. Levy $(\beta)$ is the Lévy distribution in Equation (18) and $\gamma$ is a constant rate. In the local pollination phase, the flower constancy is described by

$$
x_{i}^{t+1}=x_{i}^{t}+\varepsilon\left(x_{j}^{t}-x_{k}^{t}\right)
$$

where $x_{j}^{t}$ and $x_{k}^{t}$ are solution vectors that are transferred from different FPA flowers and $\varepsilon$ is uniformly distributed in $[0,1]$. A probability $P$ is utilized in the FPA to switch between global pollination and local 
pollination. Figure 2 summarizes the evolutionary FPA machine learning where cross-pollination and self-pollination are respectively mapped into global pollination and local pollination for FPA processes.

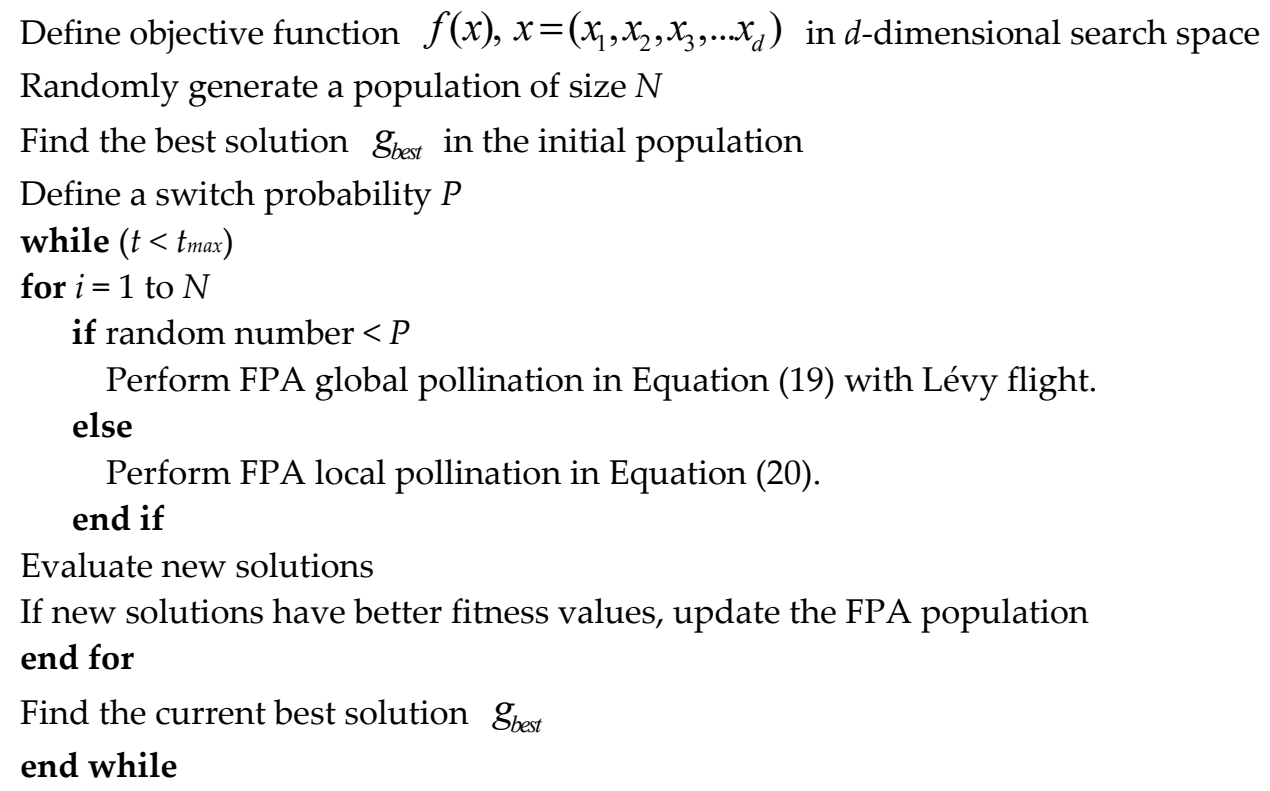

Figure 2. Pseudo code of the flower pollination algorithm (FPA) [7-9].

\subsection{FPA-Fuzzy EML Control}

When applying fuzzy systems to control applications, the fuzzy model influences the system performance. Most previous studies manually constructed the fuzzy model by using a trial-and-error approach. This subsection presents an automatic fuzzy modeling technique using FPA. With the advantages of great flexibility and robust optimization ability, the proposed FPA-fuzzy EML outperforms traditional fuzzy control schemes.

Figure 3 depicts the FPA-fuzzy EML controller, where the fuzzy model is optimized through FPA. As shown in Figure 3, the fuzzy system is composed of four parts: fuzzifier, inference engine, knowledge, and defuzzifier. The fuzzifier acts on the information in the knowledge base using membership functions. The defuzzifier performs the opposite of fuzzification, producing crisp output for a fuzzy logic system from the aggregated output of the fuzzy set. The fuzzy rule base stores the knowledge about the operation of the fuzzy process. The inference engine simulates human decisions by performing approximate reasoning. An evolutionary FPA was employed to optimize the fuzzy model, including triangular membership functions and the rule base.

In this study, the Takagi-Sugeno fuzzy model was employed to design an FPA-fuzzy EML controller. It is represented by "IF-THEN" rules with consequent parts described by linear equations, expressed by

$$
\text { Rule } i: \text { IF } x_{1} \text { is } A_{i 1}, \ldots, x_{n} \text { is } A_{\text {in }} \text { THEN } y_{i}=c_{i 0}+c_{i 1} x_{1}+\ldots+c_{i n} x_{n}
$$

where $i=1,2, \ldots, L$, and $L$ is the number of rules. The $c_{i j}(j=0,1,2, \ldots, n)$ terms are the fuzzy consequent parameters, $y_{i}$ is the output from Rule $i$, and $A_{i j}(j=0,1,2, \ldots, n)$ are fuzzy sets. Considering the input $\left(x_{1}, x_{2}, \ldots, x_{n}\right)$ in the Takagi-Sugeno fuzzy system, the final output is inferenced as follows:

$$
y=\frac{\sum_{i=1}^{L} w_{i} y_{i}}{\sum_{i=1}^{L} w_{i}}=\frac{\sum_{i=1}^{L} w_{i}\left(c_{i 0}+c_{i 1} x_{1}+\ldots+c_{i n} x_{n}\right)}{\sum_{i=1}^{L} w_{i}}=\frac{\sum_{j=1}^{n} \sum_{i=1}^{L} w_{i} c_{i j} x_{j}}{\sum_{i=1}^{L} w_{i}}
$$


where $w_{i}$ is the weight of Rule $i$, formulated as

$$
w_{i}=\prod_{j=1}^{n} A_{i j}\left(x_{j}\right)
$$

where $A_{i j}\left(x_{j}\right)$ is the grade of membership of $x_{j}$ in $A_{i j}$.



Figure 3. Architecture of the FPA-fuzzy evolutionary machine learning (EML) controller.

\subsection{Application to EML Control of Polar-Space Mecanum Vehicles}

We aimed to develop FPA-fuzzy EML control of four-wheeled Mecanum vehicles. The control gain matrices $K_{P}=\operatorname{diag}\left\{K_{p 1}, K_{p 2}, K_{p 3}\right\}$ and $K_{I}=\operatorname{diag}\left\{K_{i 1}, K_{i 2}, K_{i 3}\right\}$ were self-adjusted via the FPA-optimized fuzzy structure. Compared with traditional controllers with fixed control gains, this approach provides the heuristic information to the fuzzy control scheme in polar coordinates. The FPA fitness function was defined by including the error information in polar coordinates and the number of fuzzy rules, expressed by

$$
\text { Fitness_FPA }=\int_{0}^{t} w_{e}\left(\left(r_{e}^{M}(\tau)\right)^{2}+\left(\varphi_{e}^{M}(\tau)\right)^{2}+\left(\theta_{e}^{M}(\tau)\right)^{2}\right)+w_{l} L
$$

where $w_{e}$ and $w_{l}$ are constant weighting factors.

In the proposed FPA-fuzzy EML control scheme, triangular membership functions with center $c_{i}$ and width $w_{i}$ were utilized in the proposed FPA-fuzzy EML control system, providing the advantages of simplicity and easy implementation. An FPA individual is defined by the parameters FPA_agent $=\left\{c_{i}, w_{i}, L\right\}$. Both the numbers of rules and membership functions were considered. The initial FPA population was randomly generated, and the optimal Takagi-Sugeno fuzzy model $F P A \_$agent ${ }^{*}=\left\{c_{i}{ }^{*}, w_{i}{ }^{*}, L^{*}\right\}$ was evolved through the FPA process. The next goal was to implement an embedded cyber-physical Mecanum robotic system by including physical sensors and actuators. The proposed FPA-fuzzy cyber computation collaborates with these physical sensors/actuators to present a metaheuristic cyber-physical system.

\subsection{Embedded Cyber-Physical Mecanum Robotic System}

An embedded cyber-physical Mecanum vehicle system was developed by means of the proposed FPA-fuzzy EML technique. Figure 4 depicts the 5C architecture of the proposed metaheuristic cyber-physical system with five levels-Level 1: connection, Level 2: conversion, Level 3: cyber, Level 4: cognition, and Level 5: configuration [35-37]. This methodology integrates cyber computation 
and physical processes through sensors and actuators in a feedback loop. The proposed EML is involved in the robotic 5C structure to improve stability and performance in the Mecanum vehicles. Worthy of mention is that the embedded processor acts as the central processing unit to perform computation and conversion, thus providing an inexpensive cyber-physical robotic system.

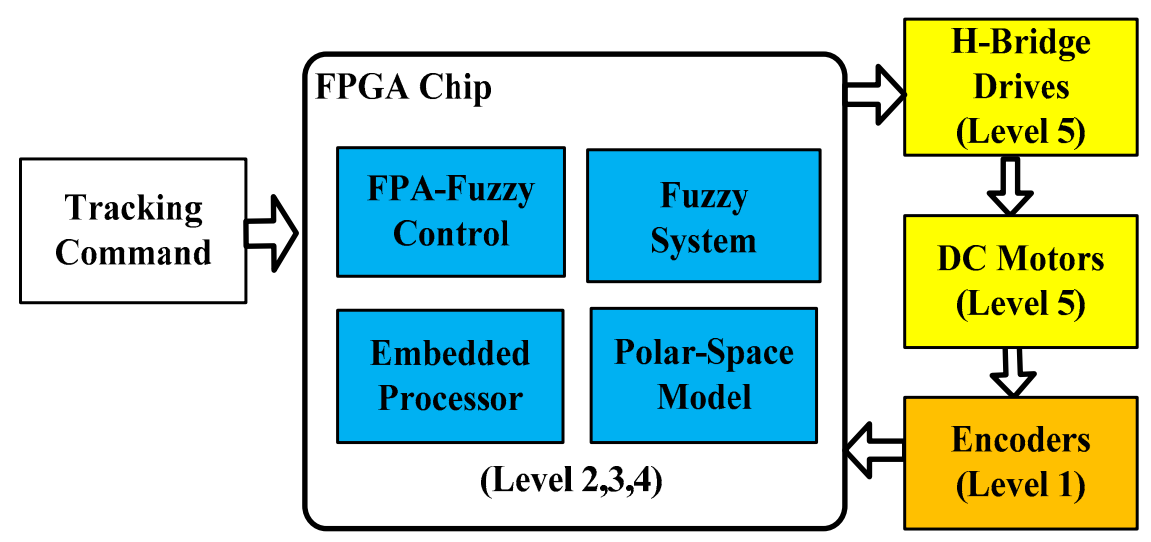

Figure 4. 5C architecture of the embedded cyber-physical Mecanum robotic system.

In the smart connection level (Level 1), the two-phase photo encoders mounted on the DC motors are used to acquire reliable data. The acquired data are then delivered to the data-to-information conversion level (Level 2), where an embedded processor was employed to perform data conversion. The acquired raw encoder data are converted into significant information on the Mecanum vehicles, including polar-space position and orientation. At the cyber level (Level 3), the polar-space kinematics model of the Mecanum vehicles and metaheuristic EML are constructed. This level receives the converted information and then performs specific analytics using the vehicle's polar-space models. The cognition level (Level 4) is the decision center of the proposed cyber-physical system in Figure 4. Taking the converted position and orientation information, the error vector of the Mecanum vehicle is obtained. The FPA-fuzzy knowledge bases are employed to generate the control decisions to achieve motion control. Finally, the configuration level (Level 5) outputs the control command to the physical omnidirectional wheels. This level behaves as a connection between the cyber world and physical world.

As shown in Figure 4, advanced robotic sensors such as two-phase photo encoders were employed in Level 1 to provide real-time data acquisition from the robotic physical world. The shaft encoders are an electro-mechanical device that converts the angular position or motion of a shaft to digital output signals. They were directly mounted on the DC motors to provide digital output about the motor's motion. A field-programmable gate array (FPGA) embedded processor was implemented in Level 2 to perform smart conversion and smart analytics in polar coordinates. This hard-core embedded ARM CPU plays a key role in Levels 2-4 to achieve real-time online control of the cyber-physical Mecanum vehicle. Finally, Level 5 drives the DC motors mounted on the Mecanum wheels to accomplish motion control tasks. H-bridges and pulse-width-modulation (PWM) custom logic were developed in this level to obtain high-accuracy motor control.

\section{Experimental Results and Discussion}

Figure 5 presents the cyber-physical Mecanum vehicle using the 5C structure and FPGA realization. To examine the polar-space tracking performance of the proposed FPA-fuzzy EML for Mecanum vehicles, the first experiment was conducted to track the polar-space desired trajectory, called the rose curve. This curve can be easily described using polar coordinates, expressed by $r_{d}^{M}(t)=40 \cos \left(4 \varphi_{d}^{M}(t)\right)+64(\mathrm{~cm})$. The initial pose of the cyber-physical Mecanum vehicle in this experiment was placed outside the curve. Figure 6 depicts the experimental result for tracking this 
special curve, and Figure 7 presents the polar-space tracking errors of orientation and position for the redundant Mecanum robotic system. These experimental results in Figures 6 and 7 validate the effectiveness of the proposed FPA-fuzzy EML control method for 5C cyber-physical Mecanum vehicles.

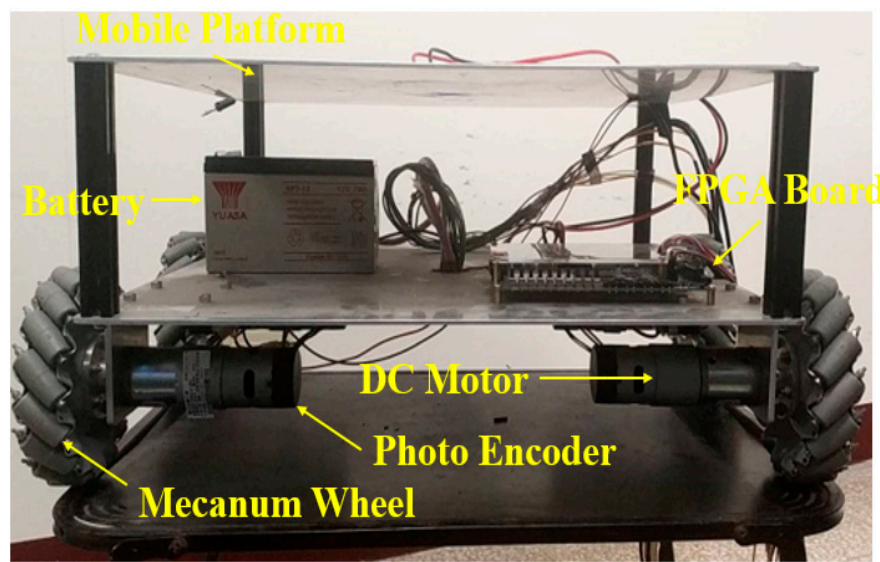

Figure 5. Cyber-physical Mecanum vehicle with FPGA realization.

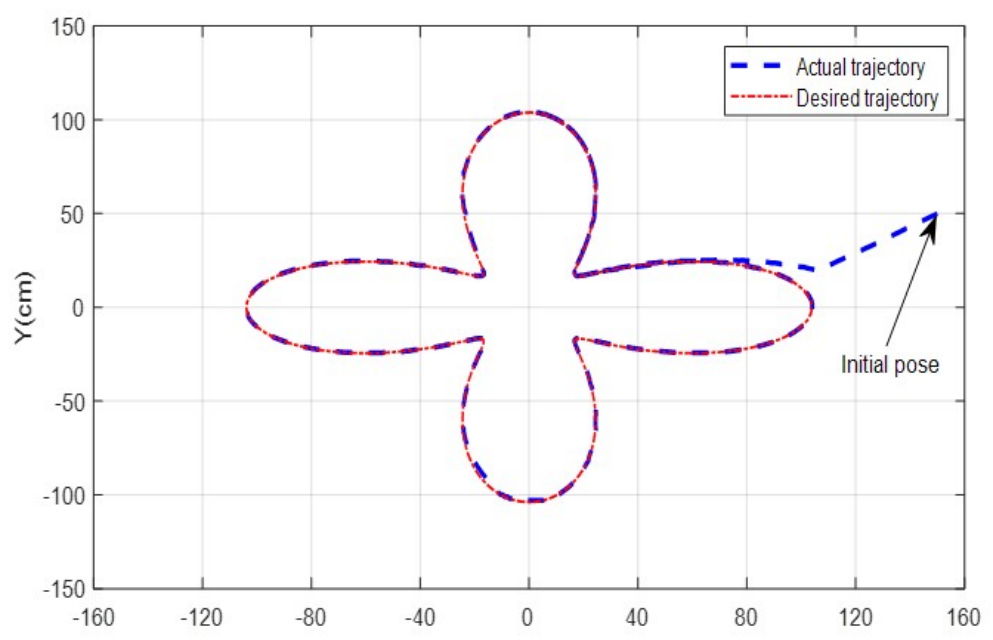

Figure 6. Experimental result of the polar-space rose curve trajectory.

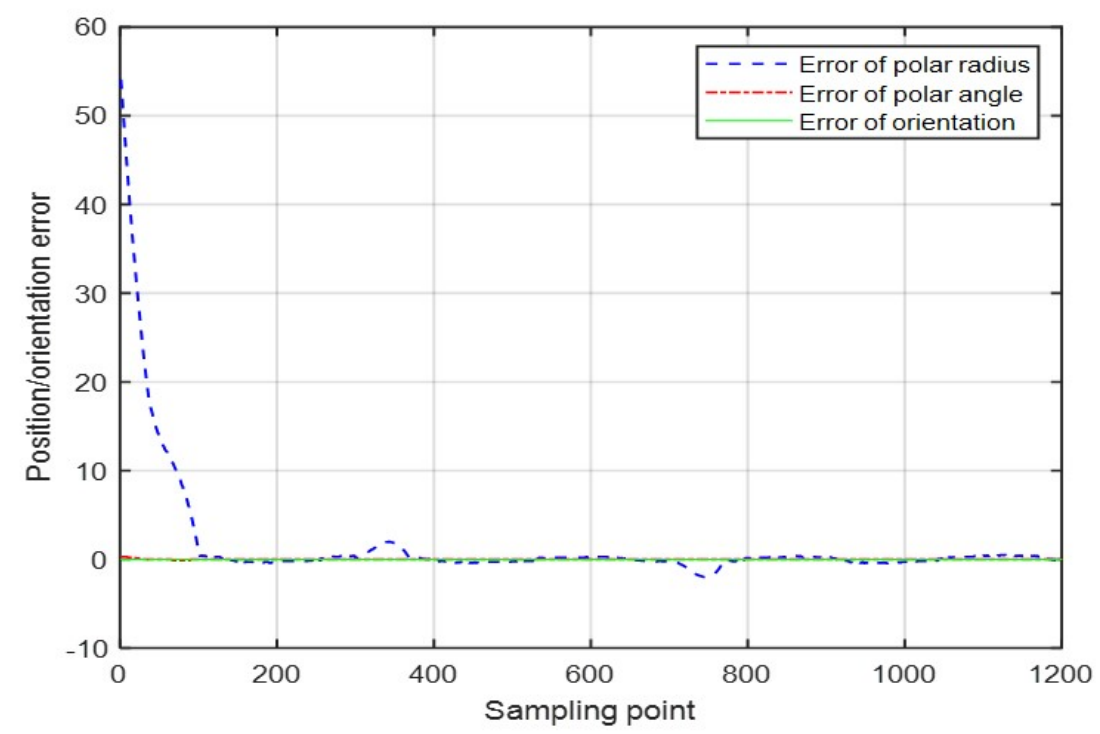

Figure 7. Tracking errors of polar-space position and orientation. 
The second experiment is provided to compare the tracking performance using Cartesian-space and polar-space control methodologies. Figure 8 presents both trajectory tracking results for the desired cardioid curve command $r_{d}^{M}(t)=150 \cos \left(\varphi_{d}^{M}(t)\right)+200$. This special trajectory can be simply described in polar coordinates. As shown in Figure 8, the polar-space FPA-fuzzy EML tracked the time-varying trajectory more quickly. More importantly, its position tracking error was less than that of the Cartesian-space controller. This comparative work clearly indicates that the proposed polar-space intelligent control scheme outperforms the traditional Cartesian-space control methods in tracking the cardioid trajectory.

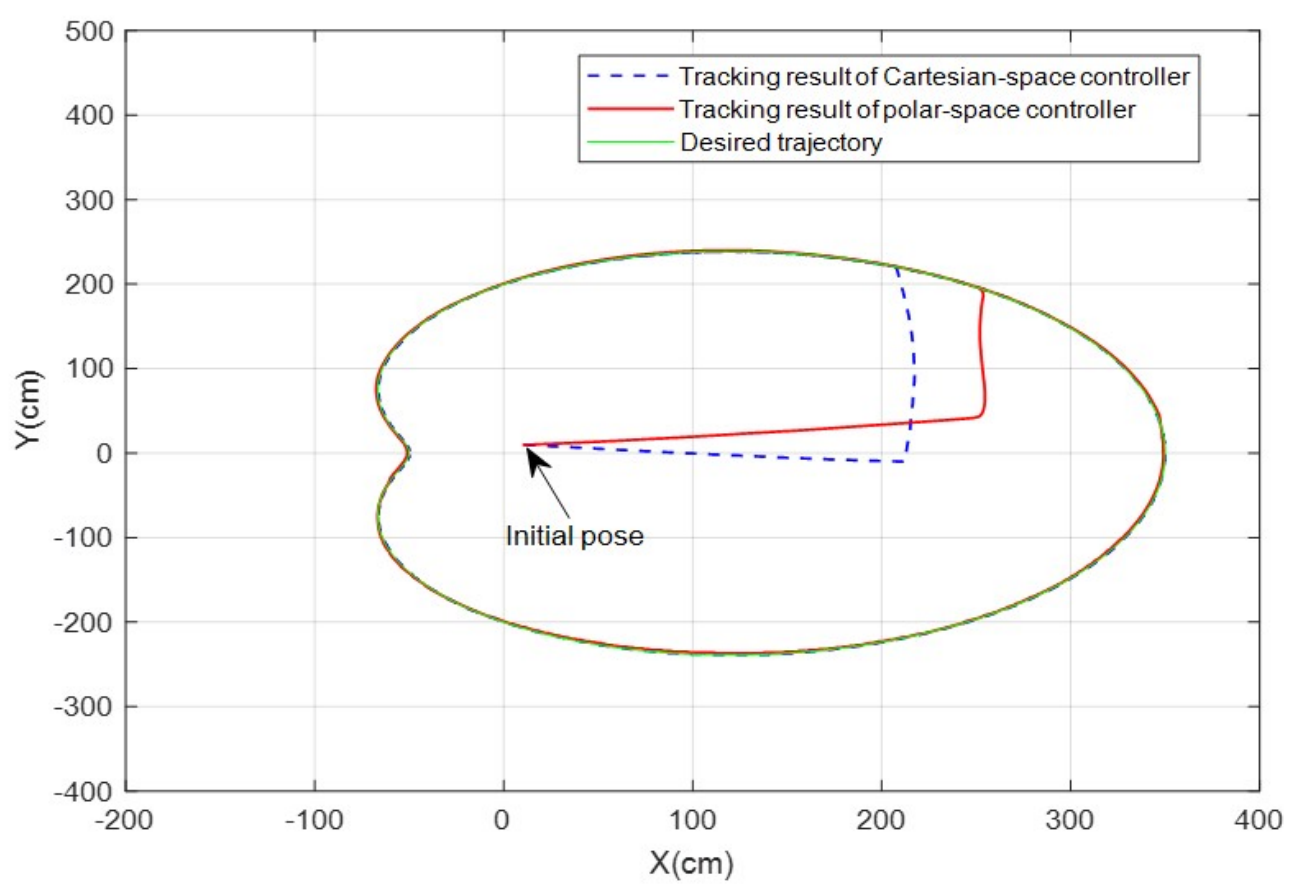

Figure 8. Cardioid trajectory tracking results in Cartesian and polar coordinates.

The third experiment was conducted to validate the effectiveness and merit of the proposed EML control method over other existing control approaches. Two modern evolutionary algorithms, $\mathrm{BA}$ and $\mathrm{ABC}$, were included to perform the same fuzzy trajectory tracking task in polar coordinates. The polar-space pose error, orientation error, and number of fuzzy rules were all considered in this study using the predefined fitness function, Equation (24). Figure 9 presents the convergent behaviors of the cardioid tracking task using BA, ABC, and FPA. This comparison analysis was performed 50 times to provide the average report. As shown in Figure 9, all three EML controllers successfully converged to a constant value, that is, they evolved optimal control parameters to drive the cyber-physical Mecanum vehicle but FPA converged the fastest. This comparative work clearly indicates that the proposed FPA-fuzzy EML control method is superior to the evolutionary BA and ABC fuzzy control systems. 


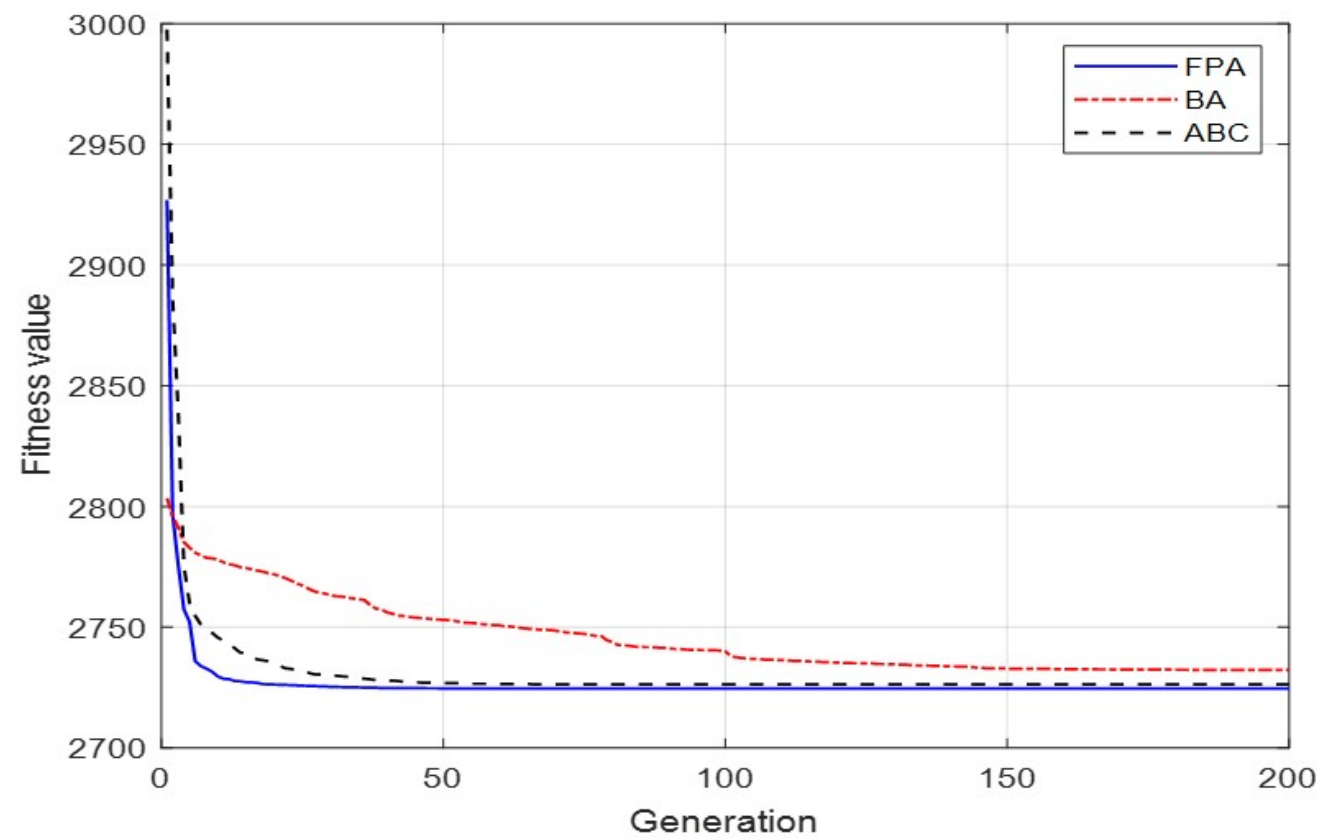

Figure 9. Convergent behaviors using the bat algorithm (BA), artificial bee colony (ABC), and FPA fuzzy control schemes.

\section{Conclusions}

In this paper, we presented an intelligent FPA-fuzzy EML control method for polar-space optimal control of cyber-physical Mecanum vehicles. The structure of the triangular membership functions and the number of fuzzy rules were optimized through the FPA evolutionary process, thus resolving the parameter tuning problem in conventional fuzzy systems. Having derived the polar-space kinematics model of Mecanum mobile vehicles, the FPA-fuzzy online control scheme was developed and its global stability was proven via Lyapunov theory. To construct the embedded cyber-physical Mecanum robotic system, an embedded processor and real-time operating system were employed. The proposed FPA-fuzzy computation collaborates with the advanced sensors and actuators of Mecanum vehicles to produce a cyber-physical robotic system using the typical 5C strategy. Finally, the FPGA-based mechatronic design and experimental setup of a Mecanum vehicle cyber-physical system was constructed. The experimental results and comparative works clearly demonstrate the effectiveness and merit of the proposed method.

Author Contributions: Conceptualization, methodology, analysis, writing-review and editing, H.-C.H.; Experiments, J.-J.X. All authors have read and agreed to the published version of the manuscript.

Funding: This research was funded by the Ministry of Science and Technology, Taiwan, under grant number MOST 109-2221-E-197-032.

Conflicts of Interest: The authors declare no conflict of interest.

\section{References}

1. Yang, B.; Zhong, L.; Zhang, X.; Shu, H.; Yu, T.; Li, H.; Jiang, L.; Sun, L. Novel bio-inspired memetic salp swarm algorithm and application to MPPT for PV systems considering partial shading condition. J. Clean. Prod. 2019, 215, 1203-1222. [CrossRef]

2. Machová, K.; Mikula, M.; Gao, X.; Mach, M. Lexicon-Based Sentiment Analysis Using Particle Swarm Optimization. Electronics 2020, 9, 1317. [CrossRef]

3. Long, W.; Wu, T.; Cai, S.; Liang, X.; Jiao, J.; Xu, M. A Novel Grey Wolf Optimizer Algorithm with Refraction Learning. IEEE Access 2019, 7, 57805-57819. [CrossRef] 
4. Cheng, R.; Jin, Y.; Narukawa, K.; Sendhoff, B. A Multiobjective Evolutionary Algorithm Using Gaussian Process-Based Inverse Modeling. IEEE Trans. Evol. Comput. 2015, 19, 838-856. [CrossRef]

5. Cecchetti, R.; De Paulis, F.; Olivieri, C.; Orlandi, A.; Buecker, M. Effective PCB Decoupling Optimization by Combining an Iterative Genetic Algorithm and Machine Learning. Electronics 2020, 9, 1243. [CrossRef]

6. Huang, H.-C.; Lin, S.-K. A Hybrid Metaheuristic Embedded System for Intelligent Vehicles Using Hypermutated Firefly Algorithm Optimized Radial Basis Function Neural Network. IEEE Trans. Ind. Inform. 2019, 15, 1062-1069. [CrossRef]

7. Yang, X.-S.; Karamanoglu, M.; He, X. Flower pollination algorithm: A novel approach for multiobjective optimization. Eng. Optim. 2013, 46, 1222-1237. [CrossRef]

8. Abdel-Basset, M.; Shawky, L.A. Flower pollination algorithm: A comprehensive review. Artif. Intell. Rev. 2019, 52, 2533-2557. [CrossRef]

9. Yang, X.-S. Flower pollination algorithm for global optimization. In Lecture Notes in Computer Science, Proceedings of the Unconventional Computing and Natural Computation: 11th International Conference, UCNC 2012, Orléan, France, 3-7 September 2012; Springer: Berlin, Germany, 2012.

10. Wang, R.; Zhou, Y. Flower Pollination Algorithm with Dimension by Dimension Improvement. Math. Probl. Eng. 2014, 2014, 1-9. [CrossRef]

11. Sharawi, M.; Emary, E.; Saroit, I.A. Flower pollination optimization algorithm for wireless sensor network lifetime global optimization. Int. J. Soft Comput. Eng. 2014, 4, 54-59.

12. Huang, C.H.; Wang, W.J.; Chiu, C.H. Design and implementation of fuzzy control on a two-wheel inverted pendulum. IEEE Trans. Ind. Electron. 2011, 58, 2988-3001. [CrossRef]

13. Cao, J.; Li, P.; Liu, H. An interval fuzzy controller for vehicle active suspension systems. IEEE Trans. Intell. Transp. Syst. 2010, 11, 885-895. [CrossRef]

14. Farrell, J.A.; Polycarpou, M.M. Adaptive Approximation Based Control: Unifying Neural, Fuzzy and Traditional Adaptive Approximation Approaches; John Wiley \& Sons: Hoboken, NJ, USA, 2006.

15. Chen, B.; Liu, X.P.; Ge, S.S.; Lin, C. Adaptive fuzzy control of a class of nonlinear systems by fuzzy approximation approach. IEEE Trans. Fuzzy Syst. 2012, 20, 1012-1021. [CrossRef]

16. Wang, L.X. Adaptive Fuzzy Systems and Control: Design and Stability Analysis; PTR Prentice Hall: Englewood Cliffs, NJ, USA, 1994.

17. Jinbao, H.; Shuai, G.; Yongyi, H.; Minglun, F. A fuzzy tension-controller based on genetic algorithm. IET Control Theory Appl. 2009, 26, 243-248.

18. Castillo, O.; Valdes, F.; Melin, P. Hierarchical genetic algorithms for topology optimization in fuzzy control systems. Int. J. Gen. Syst. 2007, 36, 575-591. [CrossRef]

19. Castillo, O.; Neyoy, H.; Soria, J.; Melin, P.; Valdez, F. A new approach for dynamic fuzzy logic parameter tuning in ant colony optimization and its application in fuzzy control of a mobile robot. Appl. Soft Comput. 2015, 28, 150-159. [CrossRef]

20. Bakdi, A.; Hentout, A.; Boutami, H.; Maoudj, A.; Hachour, O.; Bouzouia, B. Optimal path planning and execution for mobile robots using genetic algorithm and adaptive fuzzy-logic control. Robot. Auton. Syst. 2017, 89, 95-109. [CrossRef]

21. Wong, C.C.; Wang, H.Y.; Li, S.A. PSO-based motion fuzzy controller design for mobile robots. Int. J. Fuzzy Syst. 2008, 10, 284-292.

22. Pan, Y.; Er, M.J. Enhanced Adaptive Fuzzy Control with Optimal Approximation Error Convergence. IEEE Trans. Fuzzy Syst. 2013, 21, 1123-1132. [CrossRef]

23. Liu, X.; Deng, Q.-X.; Wang, Z. Design and FPGA Implementation of High-Speed, Fixed-Latency Serial Transceivers. IEEE Trans. Nucl. Sci. 2014, 61, 561-567. [CrossRef]

24. Lu, S.J.; Siqueira, P.; Vijayendra, V.; Chandrikakutty, H.; Tessier, R. Real-Time Differential Signal Phase Estimation for Space-Based Systems using FPGAs. IEEE Trans. Aerosp. Electron. Syst. 2013, 49, 1192-1209. [CrossRef]

25. Lee, C.-S.; Hsu, C.-C.; Lin, K.-E.; Chang, J.-H.; Lin, G.-H. A Project-Based Laboratory for Learning Embedded System Design with Industry Support. IEEE Trans. Educ. 2009, 53, 173-181. [CrossRef]

26. Mori, J.Y.; Arias-Garcia, J.; Sánchez-Ferreira, C.; Muñoz, D.M.; Llanos, C.H.; Motta, J.M.S.T. An FPGA-Based Omnidirectional Vision Sensor for Motion Detection on Mobile Robots. Int. J. Reconfig. Comput. 2012, 2012, 1-16. [CrossRef] 
27. Sutikno, T.; Idris, N.R.N.; Jidin, A.; Cirstea, M.N. An Improved FPGA Implementation of Direct Torque Control for Induction Machines. IEEE Trans. Ind. Inform. 2012, 9, 1280-1290. [CrossRef]

28. Idkhajine, L.; Monmasson, E.; Maalouf, A. Fully FPGA-Based Sensorless Control for Synchronous AC Drive Using an Extended Kalman Filter. IEEE Trans. Ind. Electron. 2012, 59, 3908-3918. [CrossRef]

29. Kim, J.; Woo, S.; Kim, J.; Do, J.; Kim, S.; Bae, S. Inertial navigation system for an automatic guided vehicle with Mecanum wheels. Int. J. Precis. Eng. Manuf. 2012, 13, 379-386. [CrossRef]

30. Byun, K.-S.; Song, J.-B. Design and construction of continuous alternate wheels for an omnidirectional mobile robot. J. Robot. Syst. 2003, 20, 569-579. [CrossRef]

31. Wang, Y. Motion Performance Analysis and Layout Selection for Motion System with Four Mecanum Wheels. Chin. J. Mech. Eng. 2009, 45, 307. [CrossRef]

32. Chwa, D. Sliding-Mode Tracking Control of Nonholonomic Wheeled Mobile Robots in Polar Coordinates. IEEE Trans. Control. Syst. Technol. 2004, 12, 637-644. [CrossRef]

33. Huang, H.-C. An Evolutionary Optimal Fuzzy System With Information Fusion of Heterogeneous Distributed Computing and Polar-Space Dynamic Model for Online Motion Control of Swedish Redundant Robots. IEEE Trans. Ind. Electron. 2016, 64, 1743-1750. [CrossRef]

34. Park, K.; Chung, H.; Lee, J.G. Point stabilization of mobile robots via state-space exact feedback linearization. Robot. Comput. Manuf. 2000, 16, 353-363. [CrossRef]

35. Lee, J.; Bagheri, B.; Kao, H.-A. A Cyber-Physical Systems architecture for Industry 4.0-based manufacturing systems. Manuf. Lett. 2015, 3, 18-23. [CrossRef]

36. Sheng, Y.; Lin, T.; Chen, R.; Chen, B.; Ding, G.; Xialing, X.; Xie, C.; Liu, L. Electromagnetic torque analysis-based method for performance evaluation and optimisation of closed-loop CPS regarding small signal stability. IET Cyber-Phys. Syst. Theory Appl. 2018, 3, 187-193. [CrossRef]

37. Pasqualetti, F.; Zhu, Q. Design and Operation of Secure Cyber-Physical Systems. IEEE Embed. Syst. Lett. 2014, 7, 3-6. [CrossRef]

Publisher's Note: MDPI stays neutral with regard to jurisdictional claims in published maps and institutional affiliations.

(C) 2020 by the authors. Licensee MDPI, Basel, Switzerland. This article is an open access article distributed under the terms and conditions of the Creative Commons Attribution (CC BY) license (http://creativecommons.org/licenses/by/4.0/). 